\title{
Emotional Exhaustion, Performance of Teaching Staff, and Moderation of Demographic Variables
}

\author{
${ }^{1}$ Khatiba Akhter, ${ }^{2}$ Prof. Dr. Muhammad Aslam Asghar, ${ }^{3}$ Dr. Nazir Haider Shah \\ ${ }^{1} \mathrm{PhD}$ (Education) Scholar, Mohi-Ud-Din Islamic University, Nerian Sharif, AJ\&K (Pakistan) \\ ${ }^{2,3}$ Department of Education, Mohi-Ud-Din Islamic University, Nerian Sharif, AJ\&K (Pakistan)
}

\begin{abstract}
This study was mainly aimed to explore the role of some demographic variables in the effect of emotional exhaustion on job performance of teaching staff. The study examined the direct effect of emotional exhaustion and then determined the moderating mechanism of gender, family system, and marital status. The researchers took a sample of 399 regular teachers (male $=235$, female $=164$ ) from universities and collected primary data for empirical analysis. For data collection, two questionnaires were developed on the basis of measures adopted from published and extensively followed studies. Dyadic and time lagged approaches of data collection were opted and separate questionnaires were mailed to teachers and their supervisors. Data on emotions and demographics were gathered from teachers while their performance was determined with the help of feedback from immediate supervisors, i.e. heads of the departments. For analyzing responses pertaining to direct effect, the study applied twostages structural equation modelling. Further, the study probed the moderation of gender, family system, and marital status. The results of data analysis showed a negative and significant of emotional exhaustion on performance, role, and behavior of teachers at job. Interestingly, findings did not support the significant moderation of specified demographic variables. The study observed and reported a negative effect of emotional exhaustion for respondents, irrespective of their gender, family system, and marital status. The study proposed the implications, made various suggestions, and contributed in prevailing literature of emotional exhaustion and job performance association by demonstrating the moderating mechanism of three different demographic variables.
\end{abstract}

Keywords: gender; family system; marital status; teachers; supervisors 